\title{
Statistical theory of thermodynamic and dynamic properties of the $\mathrm{RbHSO}_{4}$ ferroelectrics
}

\author{
I.R. Zachek ${ }^{1}$, R.R. Levitski ${ }^{2}$, Ya.Shchur ${ }^{12}$, O.B.Bilenk $a^{1}$ \\ ${ }^{1}$ Lviv Polytechnic National University, 12 Bandera St., 79013 Lviv, Ukraine \\ 2 Institute for Condensed Matter Physics of the National Academy of Sciences of Ukraine, \\ 1 Svientsitskii St., 79011 Lviv, Ukraine
}

Received June 10, 2015, in final form August 5, 2015

\begin{abstract}
Within the modified four-sublattice model of $\mathrm{RbHSO}_{4}$ with taking into account the piezoelectric coupling to the strains $\varepsilon_{i}, \varepsilon_{4}, \varepsilon_{5}$, and $\varepsilon_{6}$, the polarization components, static and dynamic dielectric permittivity of clamped and free crystal are calculated in the mean field approximation. At the proper choice of the values of the theory parameters, a satisfactory quantitative description of the available experimental data is obtained.
\end{abstract}

Key words: ferroelectric, dielectric permittivity, piezomodule

PACS: 77.22.Ch, 77.22.Gm, 77.65.-j, 77.80.Bh, 77.84.-s

\section{Introduction}

Chemical compounds such as sodium-potassium tartrate $\mathrm{NaKC}_{4} \mathrm{H}_{4} \mathrm{O}_{6} \cdot 4 \mathrm{H}_{2} \mathrm{O}$ (Rs), sodium-ammonium tartrate $\mathrm{NaNH}_{4} \mathrm{C}_{4} \mathrm{H}_{4} \mathrm{O}_{6} \cdot 4 \mathrm{H}_{2} \mathrm{O}$ (ARs), rubidium hydrosulphate $\mathrm{RbHSO}_{4}$ (RHS), and ammonium hydrosulphate $\mathrm{NH}_{4} \mathrm{HSO}_{4}$ (AHS) belong to the order-disorder type ferroelectrics. According to neutron and X-ray structure studies of RHS [1-4], $\mathrm{Rb}^{87}$ [5] and $\mathrm{D}^{2}$ [6, 7] NMR measurements, infrared [8] and Raman scattering experiments [9], the phase transition in RHS is of the second order. Protons are already ordered in the paraelectric phase. Only one second-order phase transition point $\left(T_{\mathrm{c}}=265 \mathrm{~K}\right)$ is present. In the high-temperature phase, the structure of RHS is monoclinic and is described by the space group $\mathrm{P} 2{ }_{1} / \mathrm{c}-$ $\mathrm{C}_{2 \mathrm{~h}}^{5}$. Below the transition point, the monoclinic symmetry remains, but the space group changes to Pc- $\mathrm{C}_{\mathrm{s}}^{2}$. The unit cell consists of eight molecules $Z=8$ in both phases.

The phase transition in RHS is associated with the motion of sulphate complexes $\left(\mathrm{SO}_{4}\right)_{11},\left(\mathrm{SO}_{4}\right)_{12}$, $\left(\mathrm{SO}_{4}\right)_{13},\left(\mathrm{SO}_{4}\right)_{14}$ between two equilibrium positions. $\left(\mathrm{SO}_{4}\right)_{25},\left(\mathrm{SO}_{4}\right)_{26},\left(\mathrm{SO}_{4}\right)_{27},\left(\mathrm{SO}_{4}\right)_{28}$-complexes are completely ordered in the entire temperature range and do not play any direct role in the ferroelectric phase transition. These complexes form a network of elementary dipoles directed along the $z$-axis (figure 1 .

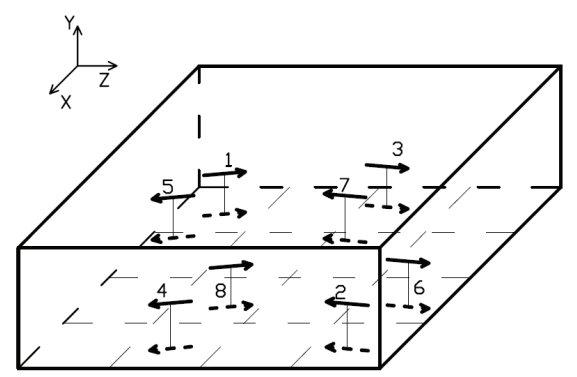

Figure 1. Orientations of the $\mathbf{d}_{q f}$ vectors within the primitive cell of $R_{s}$ in the high-symmetry phase (the paraelectric phase). 
The dipole moments created in the paraelectric phase by the (14) and (12) $\left(\mathrm{SO}_{4}\right)$ complexes have the same direction which is opposite to the direction of the dipole moments created by the (11) and (13) complexes. Analogously, the dipole moments of the (25) and (26) type complexes are opposite to those of the (27) and (28) complexes.

Two equilibrium positions (potential wells) of the $(1 f)$-complexes $(f=1,2,3,4)$ are not equivalent. Above the transition temperature $T_{\mathrm{c}}$, the $(1 f)$-complexes are located in the energetically favorable equilibrium positions (deeper potential wells). Mathematically, non-equivalence of equilibrium positions is described by an additional longitudinal field $\Delta$ acting on the dipoles of the sulphate-complexes. This field is oppositely directed for the (11), (14) and (12) complexes.

The phenomenological [10] and statistic [11,15] models of the phase transition in RHS-crystals, analogously to the Mitsui model for Rs, well describe the dielectric properties [16, 20] and the Debye-type dynamic dielectric permittivity [12-15] in the mean field approximation. However, it is impossible to calculate the experimentally measurable dielectric permittivity of a mechanically free crystal, the piezoelectric coefficients, elastic constants and transverse dielectric permittivities using these models [12,15]. That is why the piezoelectric coupling to the strains should be taken into account [21].

In this work we propose a modification of the four-sublattice model of the $\mathrm{RbHSO}_{4}$ crystals, which takes into account the piezoelectric coupling to the strains $\varepsilon_{i}, \varepsilon_{j}$ in the ferroelectric phase. The dielectric, piezoelectric, elastic, thermal and dynamic characteristics of RHS are calculated within the mean field approximation. The corresponding experimental data for this crystal are described.

\section{Four-sublattice model: Hamiltonian}

The system Hamiltonian is a modification of the Hamiltonian proposed in [22] that takes into account the piezoelectric coupling, and a generalization of the Hamiltonian in [13] to the 'three-dimensional' model. In the quasi-spin representation, it reads as follows:

$$
\begin{aligned}
\hat{H}= & N U_{\text {seed }}-\frac{1}{2} \sum_{q q^{\prime}} \sum_{f=1}^{4} J_{f f}\left(q q^{\prime}\right) \frac{\sigma_{q f}}{2} \frac{\sigma_{q^{\prime} f}}{2}-\frac{1}{2} \sum_{q q^{\prime}} \sum_{f \neq f^{\prime}} K_{f f^{\prime}}\left(q q^{\prime}\right) \frac{\sigma_{q f}}{2} \frac{\sigma_{q^{\prime} f^{\prime}}}{2} \\
& -\Delta \sum_{q}\left(-\frac{\sigma_{q 1}}{2}-\frac{\sigma_{q 2}}{2}+\frac{\sigma_{q 3}}{2}+\frac{\sigma_{q 4}}{2}\right)-\mu_{1} E_{1} \sum_{q}\left(\frac{\sigma_{q 1}}{2}-\frac{\sigma_{q 2}}{2}+\frac{\sigma_{q 3}}{2}-\frac{\sigma_{q 4}}{2}\right) \\
& -\mu_{2} E_{2} \sum_{q}\left(-\frac{\sigma_{q 1}}{2}+\frac{\sigma_{q 2}}{2}+\frac{\sigma_{q 3}}{2}-\frac{\sigma_{q 4}}{2}\right)-\mu_{3} E_{3} \sum_{q}\left(\frac{\sigma_{q 1}}{2}+\frac{\sigma_{q 2}}{2}+\frac{\sigma_{q 3}}{2}+\frac{\sigma_{q 4}}{2}\right),
\end{aligned}
$$

where $N$ is the number of unit cells. In 2.1) $J_{f f^{\prime}}\left(q q^{\prime}\right)$ and $K_{f f^{\prime}}\left(q q^{\prime}\right)$ are the interaction potentials between the pseudospins from the same and from different chains, respectively; the parameter $\Delta$ describes the asymmetry of the potential, in which the pseudospins move; $\mu_{i}$ is the effective dipole moment per one pseudospin; $\sigma_{q f}$ is the $z$-component of the pseudospin operator situated at $f$-th bond $(f=1,2,3,4)$ in $q$-th unit cell.

$U_{1 \text { seed }}$ is the seed energy that includes the elastic, piezoelectric and dielectric contributions expressed in terms of the electric fields $E_{i}(i=1,2,3)$ and strains $\varepsilon_{i}$ and $\varepsilon_{j}(j=i+3) . c_{j j}^{E 0}(T), e_{i j}^{0}, \chi_{i i}^{\varepsilon 0}$ are the so-called seed elastic and piezoelectric constants and dielectric permittivities:

$$
\begin{aligned}
U_{\text {seed }}=v[ & \frac{1}{2} \sum_{i, i^{\prime}=1}^{3} c_{i i^{\prime}}^{E 0}(T) \varepsilon_{i} \varepsilon_{i^{\prime}}+\frac{1}{2} \sum_{j=4}^{6} c_{j j}^{E 0}(T) \varepsilon_{j}^{2}-\sum_{i=1}^{3} e_{3 i}^{0} \varepsilon_{i} E_{3}-e_{35}^{0} \varepsilon_{5} E_{3} \\
& \left.-\frac{1}{2} \chi_{11}^{\varepsilon 0} E_{1}^{2}-\frac{1}{2} \chi_{22}^{\varepsilon 0} E_{2}^{2}-\frac{1}{2} \chi_{33}^{\varepsilon 0} E_{3}^{2}\right]
\end{aligned}
$$

$v$ is the unit cell volume.

Having analyzed the results of [23], we assume the seed elastic constants $c_{j j}^{E 0}(T)$ to be linearly decreasing with temperature [24]

$$
c_{i i^{\prime}}^{E 0}(T)=c_{i i^{\prime}}^{E 0}-k_{i i^{\prime}}\left(T-T_{\mathrm{c}}\right), \quad c_{j j}^{E 0}(T)=c_{j j}^{E 0}-k_{j j}\left(T-T_{\mathrm{c}}\right),
$$


where the coefficients $k_{j j}$ phenomenologically take into account the high-temperature lattice anharmonism.

We make an identity transformation

$$
\sigma_{q f}=\eta_{f}+\left(\sigma_{q f}-\eta_{f}\right), \quad(f=1,2,3,4),
$$

and neglect the quadric fluctuations. The Fourier transforms of the interaction constants at $\mathbf{q}=0, J=$ $J_{f f}=\sum_{q^{\prime}} J_{f f}\left(q q^{\prime}\right), K_{f f^{\prime}}=\sum_{q^{\prime}} K_{f f^{\prime}}\left(q q^{\prime}\right)$ and $\Delta$ are expanded in series over the strains $\varepsilon_{i}, \varepsilon_{j}$ up to the linear terms:

$$
\begin{array}{ll}
J=J^{0}+\frac{\partial J}{\partial \varepsilon_{i}} \varepsilon_{i}=J^{0}+\sum_{i=1}^{3} \psi_{1 i} \varepsilon_{i}+\sum_{j=4}^{6} \psi_{1 j} \varepsilon_{j}, & K_{12}=K_{12}^{0}+\sum_{i=1}^{3} \psi_{2 i} \varepsilon_{i}+\sum_{j=4}^{6} \psi_{2 j} \varepsilon_{j} \\
K_{13}=K_{13}^{0}+\sum_{i=1}^{3} \psi_{3 i} \varepsilon_{i}+\sum_{j=4}^{6} \psi_{3 j} \varepsilon_{j}, & K_{14}=K_{14}^{0}+\sum_{i=1}^{3} \psi_{4 i} \varepsilon_{i}+\sum_{j=4}^{6} \psi_{4 j} \varepsilon_{j} \\
\Delta=\Delta^{0}+\sum_{i=1}^{3} \psi_{5 i} \varepsilon_{i}+\sum_{j=4}^{6} \psi_{5 j} \varepsilon_{j} . &
\end{array}
$$

As a result, Hamiltonian (2.1) within the mean field approximation takes the form:

$$
\begin{aligned}
H^{(0)}= & v U_{\text {seed }}+\frac{1}{8} J^{0}\left(\eta_{1}^{2}+\eta_{2}^{2}+\eta_{3}^{2}+\eta_{4}^{2}\right) \\
& +\frac{1}{4} K_{12}^{0}\left(\eta_{1} \eta_{2}+\eta_{3} \eta_{4}\right)+\frac{1}{4} K_{13}^{0}\left(\eta_{1} \eta_{3}+\eta_{2} \eta_{4}\right)+\frac{1}{4} K_{14}^{0}\left(\eta_{1} \eta_{4}+\eta_{2} \eta_{3}\right) \\
+ & \frac{1}{8}\left(\sum_{i=1}^{3} \psi_{1 i} \varepsilon_{i}+\sum_{j=4}^{6} \psi_{1 j} \varepsilon_{j}\right)\left(\eta_{1}^{2}+\eta_{2}^{2}+\eta_{3}^{2}+\eta_{4}^{2}\right)+\frac{1}{4}\left(\sum_{i=1}^{3} \psi_{2 i} \varepsilon_{i}+\sum_{j=4}^{6} \psi_{2 j} \varepsilon_{j}\right)\left(\eta_{1} \eta_{2}+\eta_{3} \eta_{4}\right) \\
+ & \frac{1}{4}\left(\sum_{i=1}^{3} \psi_{3 i} \varepsilon_{i}+\sum_{j=4}^{6} \psi_{3 j} \varepsilon_{j}\right)\left(\eta_{1} \eta_{3}+\eta_{2} \eta_{4}\right)+\frac{1}{4}\left(\sum_{i=1}^{3} \psi_{4 i} \varepsilon_{i}+\sum_{j=4}^{6} \psi_{4 j} \varepsilon_{j}\right)\left(\eta_{1} \eta_{4}+\eta_{2} \eta_{3}\right), \\
\hat{H}_{s}= & -\sum_{q}\left(\mathscr{H}_{1} \frac{\sigma_{q 1}}{2}+\mathscr{H}_{2} \frac{\sigma_{q 2}}{2}+\mathscr{H}_{3} \frac{\sigma_{q 3}}{2}+\mathscr{H}_{4} \frac{\sigma_{q 4}}{2}\right) .
\end{aligned}
$$

Thus, we find the mean pseudospin values

$$
\eta_{f}=\tanh \frac{\beta}{2} \mathscr{H}_{f}
$$

Let us use new variables:

$$
\begin{aligned}
& \xi_{1}=\frac{1}{4}\left(-\eta_{1}-\eta_{2}+\eta_{3}+\eta_{4}\right)=\frac{1}{4}\left(-\tanh \frac{\beta}{2} \mathscr{H}_{1}-\tanh \frac{\beta}{2} \mathscr{H}_{2}+\tanh \frac{\beta}{2} \mathscr{H}_{3}+\tanh \frac{\beta}{2} \mathscr{H}_{4}\right), \\
& \xi_{2}=\frac{1}{4}\left(-\eta_{1}+\eta_{2}+\eta_{3}-\eta_{4}\right)=\frac{1}{4}\left(-\tanh \frac{\beta}{2} \mathscr{H}_{1}+\tanh \frac{\beta}{2} \mathscr{H}_{2}+\tanh \frac{\beta}{2} \mathscr{H}_{3}-\tanh \frac{\beta}{2} \mathscr{H}_{4}\right), \\
& \xi_{3}=\frac{1}{4}\left(\eta_{1}+\eta_{2}+\eta_{3}+\eta_{4}\right)=\frac{1}{4}\left(\tanh \frac{\beta}{2} \mathscr{H}_{1}+\tanh \frac{\beta}{2} \mathscr{H}_{2}+\tanh \frac{\beta}{2} \mathscr{H}_{3}+\tanh \frac{\beta}{2} \mathscr{H}_{4}\right), \\
& \zeta=\frac{1}{4}\left(\eta_{1}-\eta_{2}+\eta_{3}-\eta_{4}\right)=\frac{1}{4}\left(\tanh \frac{\beta}{2} \mathscr{H}_{1}-\tanh \frac{\beta}{2} \mathscr{H}_{2}+\tanh \frac{\beta}{2} \mathscr{H}_{3}-\tanh \frac{\beta}{2} \mathscr{H}_{4}\right),
\end{aligned}
$$

where the self-consistency fields $\mathscr{H}_{f}$ are given by the expressions:

$$
\begin{array}{ll}
\mathscr{H}_{1}=\left(-\gamma_{1}-\gamma_{2}+\gamma_{3}+\delta\right), & \mathscr{H}_{2}=\left(-\gamma_{1}+\gamma_{2}+\gamma_{3}-\delta\right), \\
\mathscr{H}_{3}=\left(\gamma_{1}+\gamma_{2}+\gamma_{3}+\delta\right), & \mathscr{H}_{4}=\left(\gamma_{1}-\gamma_{2}+\gamma_{3}-\delta\right),
\end{array}
$$

and

$$
\gamma_{1}=\left(\frac{J_{1}}{2} \xi_{1}+\mu_{1} E_{1}\right), \quad \gamma_{2}=\left(\frac{J_{2}}{2} \xi_{2}+\mu_{2} E_{2}\right), \quad \gamma_{3}=\left(\frac{J_{3}}{2} \xi_{3}+\mu_{3} E_{3}\right), \quad \delta=\left(\frac{J_{4}}{2} \zeta+\Delta\right) .
$$


Taking into account [2.3), we obtain

$$
\begin{array}{ll}
J_{1}=J_{1}^{0}+\sum_{i=1}^{3} \bar{\psi}_{1 i} \varepsilon_{i}+\sum_{j=4}^{6} \bar{\psi}_{1 j} \varepsilon_{j}, & J_{2}=J_{2}^{0}+\sum_{i=1}^{3} \bar{\psi}_{2 i} \varepsilon_{i}+\sum_{j=4}^{6} \bar{\psi}_{2 j} \varepsilon_{j}, \\
J_{3}=J_{3}^{0}+\sum_{i=1}^{3} \bar{\psi}_{3 i} \varepsilon_{i}+\sum_{j=4}^{6} \bar{\psi}_{3 j} \varepsilon_{j}, & J_{4}=J_{4}^{0}+\sum_{i=1}^{3} \bar{\psi}_{4 i} \varepsilon_{i}+\sum_{j=4}^{6} \bar{\psi}_{4 j} \varepsilon_{j}, \\
\Delta & =\Delta^{0}+\sum_{i=1}^{3} \psi_{5 i} \varepsilon_{i}+\sum_{j=4}^{6} \psi_{5 j} \varepsilon_{j},
\end{array}
$$

where

$$
\begin{array}{cc}
J_{1}^{0}=-J^{0}-K_{12}^{0}+K_{13}^{0}+K_{14}^{0}, & J_{2}^{0}=J^{0}-K_{12}^{0}-K_{13}^{0}+K_{14}^{0}, \\
J_{3}^{0}=J^{0}+K_{12}^{0}+K_{13}^{0}+K_{14}^{0}, & J_{4}^{0}=J^{0}-K_{12}^{0}+K_{13}^{0}-K_{14}^{0}, \\
\bar{\psi}_{1 i}=-\psi_{1 i}-\psi_{2 i}+\psi_{3 i}+\psi_{4 i}, & \bar{\psi}_{1 j}=-\psi_{1 j}-\psi_{2 j}+\psi_{3 j}+\psi_{4 j}, \\
\bar{\psi}_{2 i}=\psi_{1 i}-\psi_{2 i}-\psi_{3 i}+\psi_{4 i}, & \bar{\psi}_{2 j}=\psi_{1 j}-\psi_{2 j}-\psi_{3 j}+\psi_{4 j}, \\
\bar{\psi}_{3 i}=\psi_{1 i}+\psi_{2 i}+\psi_{3 i}+\psi_{4 i}, & \bar{\psi}_{35}=\psi_{15}+\psi_{25}+\psi_{35}+\psi_{45}, \\
\bar{\psi}_{4 i}=\psi_{1 i}-\psi_{2 i}+\psi_{3 i}-\psi_{4 i}, & \bar{\psi}_{45}=\psi_{15}-\psi_{25}+\psi_{35}-\psi_{45} .
\end{array}
$$

Parameters $\xi_{1}, \xi_{2}, \xi_{3}$ describe the dipole pseudospin ordering along the $a, b$ and $c$-axes, respectively, and the parameter $\zeta$ is responsible for the paraelectric phase pseudospin ordering.

Without external electric fields and mechanical strains, the pseudospin mean values in the paraelectric phases are $\eta_{1}=-\eta_{2}=\eta_{3}=-\eta_{4}=\eta$ and $\xi_{1 p}=\xi_{2 p}=\xi_{3 p}=0$, respectively, and

$$
\zeta_{p}=\tanh \frac{\beta}{2}\left(\frac{J_{4}}{2} \zeta_{p}+\Delta\right)
$$

In the ferroelectric phase at zero fields $E_{i}=0$ and stresses $\sigma_{j}=0, \eta_{1}=\eta_{3}=\eta_{13}, \eta_{2}=\eta_{4}=\eta_{24}$. As a result $\xi_{1 s}=0, \xi_{2 s}=0$, and

$$
\begin{aligned}
\xi_{3 s} & =\frac{1}{2}\left[\tanh \frac{\beta}{2}\left(\frac{J_{3}}{2} \xi_{1 s}+\frac{J_{4}}{2} \zeta_{s}+\Delta\right)+\tanh \frac{\beta}{2}\left(\frac{J_{3}}{2} \xi_{1 s}-\frac{J_{4}}{2} \zeta_{s}-\Delta\right)\right], \\
\zeta_{s} & =\frac{1}{2}\left[\tanh \frac{\beta}{2}\left(\frac{J_{3}}{2} \xi_{1 s}+\frac{J_{4}}{2} \zeta_{s}+\Delta\right)-\tanh \frac{\beta}{2}\left(\frac{J_{3}}{2} \xi_{1 s}-\frac{J_{4}}{2} \zeta_{s}-\Delta\right)\right] .
\end{aligned}
$$

\section{Thermodynamic characteristics of RHS}

To calculate the dielectric, piezoelectric, and elastic characteristics RHS, we use the electric thermodynamical potential per unit cell obtained in the mean field approximation

$$
\begin{aligned}
g=\frac{G}{N}= & \nu U_{\text {seed }}-4 \frac{1}{\beta} \ln 2-\frac{1}{\beta} \sum_{f=1}^{4} \ln \cosh \frac{\beta}{2} \mathscr{H}_{f} \\
& +\frac{1}{2}\left(J_{1}^{0}+\sum_{i=1}^{3} \overline{\psi_{1 i}} \varepsilon_{i}+\sum_{j=4}^{6} \overline{\psi_{1 j}} \varepsilon_{j}\right) \xi_{1}^{2}+\frac{1}{2}\left(J_{2}^{0}+\sum_{i=1}^{3} \bar{\psi}_{2 i} \varepsilon_{i}+\sum_{j=4}^{6} \overline{\psi_{2 j}} \varepsilon_{j}\right) \xi_{2}^{2} \\
& +\frac{1}{2}\left(J_{3}^{0}+\sum_{i=1}^{3} \overline{\psi_{3 i}} \varepsilon_{i}+\sum_{j=4}^{6} \overline{\psi_{3 j}} \varepsilon_{j}\right) \xi_{3}^{2}+\frac{1}{2}\left(J_{4}^{0}+\sum_{i=1}^{3} \bar{\psi}_{4 i} \varepsilon_{i}+\sum_{j=4}^{6} \overline{\psi_{4 j}} \varepsilon_{j}\right) \zeta^{2} .
\end{aligned}
$$

From the thermodynamic equilibrium conditions

$$
\frac{1}{v}\left(\frac{\partial g}{\partial \varepsilon_{i}}\right)_{E_{i}}=0, \quad \frac{1}{v}\left(\frac{\partial g}{\partial \varepsilon_{j}}\right)_{E_{i}, \sigma_{i}}=0, \quad \frac{1}{v}\left(\frac{\partial g}{\partial E_{i}}\right)=-P_{i}
$$


we obtain

$$
\begin{aligned}
& 0=c_{1 i}^{E 0}(T) \varepsilon_{1}+c_{i 2}^{E 0}(T) \varepsilon_{2}+c_{i 3}^{E 0}(T) \varepsilon_{3}-e_{3 i}^{0} E_{3}-\frac{\overline{\psi_{1 i}}}{2 v} \xi_{1}^{2}-\frac{\overline{\psi_{2 i}}}{2 v} \xi_{2}^{2}-\frac{\overline{\psi_{3 i}}}{2 v} \xi_{3}^{2}-\frac{\overline{\psi_{4 i}}}{2 v} \zeta^{2}-\frac{2 \psi_{5 i}}{v} \zeta, \\
& 0=c_{44}^{E 0}(T) \varepsilon_{4}-\frac{\overline{\psi_{14}}}{2 v} \xi_{1}^{2}-\frac{\overline{\psi_{24}}}{2 v} \xi_{2}^{2}-\frac{\overline{\psi_{34}}}{2 v} \xi_{3}^{2}-\frac{\overline{\psi_{44}}}{2 v} \zeta^{2}-\frac{2 \psi_{54}}{v} \zeta, \\
& 0=c_{55}^{E 0}(T) \varepsilon_{5}-e_{35}^{0} E_{3}-\frac{\overline{\psi_{15}}}{2 v} \xi_{1}^{2}-\frac{\overline{\psi_{25}}}{2 v} \xi_{2}^{2}-\frac{\overline{\psi_{35}}}{2 v} \xi_{3}^{2}-\frac{\overline{\psi_{45}}}{2 v} \zeta^{2}-\frac{2 \psi_{55}}{v} \zeta \\
& 0=c_{66}^{E 0}(T) \varepsilon_{6}-\frac{\overline{\psi_{16}}}{2 v} \xi_{1}^{2}-\frac{\overline{\psi_{26}}}{2 v} \xi_{2}^{2}-\frac{\overline{\psi_{36}}}{2 v} \xi_{3}^{2}-\frac{\overline{\psi_{46}}}{2 v} \zeta^{2}-\frac{2 \psi_{56}}{v} \zeta \\
& P_{1}=e_{11}^{0} \varepsilon_{1}+e_{12}^{0} \varepsilon_{2}+e_{13}^{0} \varepsilon_{3}+e_{15}^{0} \varepsilon_{5}+\chi_{11}^{\varepsilon 0} E_{1}+\frac{2 \mu_{1}}{v} \xi_{1}, \\
& P_{2}=e_{24}^{0} \varepsilon_{4}+e_{26}^{0} \varepsilon_{6}+\chi_{22}^{\varepsilon 0} E_{2}+\frac{2 \mu_{2}}{v} \xi_{2}, \\
& P_{3}=e_{31}^{0} \varepsilon_{1}+e_{32}^{0} \varepsilon_{2}+e_{33}^{0} \varepsilon_{3}+e_{35}^{0} \varepsilon_{5}+\chi_{33}^{\varepsilon 0} E_{3}+\frac{2 \mu_{3}}{v} \xi_{3} .
\end{aligned}
$$

In the ferroelectric phase, the static isothermic dielectric permittivities of mechanically clamped RHS along the crystallographic axes are as follows:

$$
\chi_{i i s}^{T \varepsilon}(0)=\lim _{E_{i} \rightarrow 0}\left(\frac{\partial P_{i}}{\partial E_{i}}\right)_{\varepsilon_{j}}=\chi_{i i}^{\varepsilon 0}+\frac{\mu_{i}^{2}}{v} \beta F_{1 i s}(0) .
$$

The following notations are used

$$
\begin{aligned}
& F_{11 s}(0)=\frac{\rho_{31}-\left(\rho_{31}^{2}-\rho_{32}^{2}\right) \frac{\beta J_{2}}{4}}{1-\rho_{31}\left(\frac{\beta J_{1}}{4}+\frac{\beta J_{2}}{4}\right)+\left(\rho_{31}^{2}-\rho_{32}^{2}\right) \frac{\beta J_{1}}{4} \frac{\beta J_{2}}{4}}, \\
& F_{12 s}(0)=\frac{\rho_{31}-\left(\rho_{31}^{2}-\rho_{32}^{2}\right) \frac{\beta J_{1}}{4}}{1-\rho_{31}\left(\frac{\beta J_{1}}{4}+\frac{\beta J_{2}}{4}\right)+\left(\rho_{31}^{2}-\rho_{32}^{2}\right) \frac{\beta J_{1}}{4} \frac{\beta J_{2}}{4},} \\
& F_{13 s}(0)=\frac{\rho_{31}-\left(\rho_{31}^{2}-\rho_{32}^{2}\right) \frac{\beta J_{4}}{4}}{1-\rho_{31}\left(\frac{\beta J_{3}}{4}+\frac{\beta J_{4}}{4}\right)+\left(\rho_{31}^{2}-\rho_{32}^{2}\right) \frac{\beta J_{3}}{4} \frac{\beta J_{4}}{4}},
\end{aligned}
$$

and

In the paraelectric phase:

$$
\rho_{31}=1-\xi_{3 s}^{2}-\zeta_{s}^{2}, \quad \rho_{32}=2 \xi_{3 s} \zeta_{s}
$$

$$
\chi_{i i p}^{T \varepsilon}(0)=\chi_{i i}^{\varepsilon 0}+\frac{\mu_{i}^{2}}{v} \beta F_{1 i p}(0), \quad(i=1,2,3)
$$

where

$$
F_{1 i p}(0)=\frac{1-\zeta_{p}^{2}}{1-\left(1-\zeta_{p}^{2}\right) \frac{\beta J_{i}}{4}} .
$$

From relations [3.3, we get expressions for isothermic piezoelectric coefficients $e_{i j}$ of RHS

$$
\begin{aligned}
& e_{3 i s}^{T}=\left(\frac{\partial P_{3}}{\partial \varepsilon_{i}}\right)_{E_{3}}=e_{3 i}^{0}+\frac{\mu_{3}}{v} \frac{\beta}{2}\left[\bar{\psi}_{3 i} \xi_{3 s} F_{13 s}(0)-\left(\bar{\psi}_{4 i} \zeta_{s}+2 \psi_{5 i}\right) \bar{F}_{13 s}(0)\right], \\
& e_{35 s}^{T}=\left(\frac{\partial P_{3}}{\partial \varepsilon_{5}}\right)_{E_{3}}=e_{35}^{0}+\frac{\mu_{3}}{v} \frac{\beta}{2}\left[\bar{\psi}_{35} \xi_{3 s} F_{13 s}(0)-\left(\bar{\psi}_{45} \zeta_{s}+2 \psi_{55}\right) \bar{F}_{13 s}(0)\right] .
\end{aligned}
$$

By differentiating the relations 3.3 with respect to the strains at a constant polarization, we obtain the expressions for the for piezoelectric constants

$$
h_{3 i s}^{T}=\frac{e_{3 i s}}{\chi_{33 s}^{\varepsilon}}, \quad h_{35 s}^{T}=\frac{e_{35 s}}{\chi_{33 s}^{\varepsilon}} .
$$


Now, we calculate the contributions of the pseudospin system to the elastic constants of RHS. From (3.2) we obtain the relations for elastic coefficients at a constant field:

$$
\begin{aligned}
& c_{i i^{\prime} s}^{T E}=\left(\frac{\partial \sigma_{i}}{\partial \varepsilon_{i^{\prime}}}\right)_{E_{i}}=c_{i i^{\prime}}^{E 0}(T)-\frac{\beta \overline{\psi_{3 i}} \overline{\psi_{3 i^{\prime}}}}{4 v} \xi_{3 s}^{2} F_{13 s}(0)-\frac{\beta\left(\overline{\psi_{4 i}} \zeta_{s}+2 \psi_{5 i}\right)\left(\overline{\psi_{4 i^{\prime}}} \zeta_{s}+2 \psi_{5 i^{\prime}}\right)}{4 v} F_{14 s}(0) \\
& +\frac{\beta\left(\overline{\left.\psi_{3 i} \psi_{4 i^{\prime}}+{\overline{\psi_{3 i}}}^{-} \bar{\psi}_{4 i}\right)}\right.}{4 v} \xi_{3 s} \zeta_{s} \bar{F}_{13 s}(0)+\frac{\beta\left(\overline{\psi_{4 i}} \psi_{5 i^{\prime}}+\overline{\psi_{4 i^{\prime}}} \psi_{5 i}\right)}{2 v} \xi_{3 s} \bar{F}_{13 s}(0), \\
& c_{i i^{\prime} p}^{T E}=c_{i i^{\prime}}^{E 0}(T)-\frac{\beta\left(\bar{\psi}_{4 i} \zeta_{p}+2 \psi_{5 i}\right)\left(\bar{\psi}_{4 i^{\prime}} \zeta_{p}+2 \psi_{5 i^{\prime}}\right)}{4 v} F_{14 p}(0), \\
& c_{j j s}^{T E}=\left(\frac{\partial \sigma_{j}}{\partial \varepsilon_{j}}\right)_{E_{i}}=c_{j j}^{E 0}(T)-\frac{\beta \bar{\psi}_{3 j}^{2}}{4 v} \xi_{3 s}^{2} F_{13 s}(0)-\frac{\beta\left(\overline{\psi_{4 j}} \zeta_{s}+2 \psi_{5 j}\right)^{2}}{4 v} F_{14 s}(0) \\
& +\frac{\beta \overline{\psi_{3 j}} \overline{\psi_{4 j}}}{2 v} \xi_{3 s} \zeta_{s} \bar{F}_{13 s}(0)+\frac{\beta \overline{\psi_{3 j}} \psi_{5 j}}{v} \xi_{3 s} \bar{F}_{13 s}(0) \\
& c_{j j p}^{T E}=c_{j j}^{E 0}(T)-\frac{\beta\left(\overline{\psi_{4 j}} \zeta_{s}+2 \psi_{5 j}\right)^{2}}{4 v} F_{14 p}(0),
\end{aligned}
$$

where

$$
F_{14 s}(0)=\frac{\rho_{31}-\left(\rho_{31}^{2}-\rho_{32}^{2}\right) \frac{\beta J_{3}}{4}}{1-\rho_{31}\left(\frac{\beta J_{3}}{4}+\frac{\beta J_{4}}{4}\right)+\left(\rho_{31}^{2}-\rho_{32}^{2}\right) \frac{\beta J_{3}}{4} \frac{\beta J_{4}}{4}}, \quad F_{14 p}(0)=\frac{1-\zeta_{p}^{2}}{1-\left(1-\zeta_{p}^{2}\right) \frac{\beta J_{4}}{4}} .
$$

From (3.2), 3.3 we get isothermic coefficients of piezoelectric strain $d_{1 i}=\left(\partial P_{1} / \partial \sigma_{i}\right)_{E_{1}}$, $d_{i j}=\left(\partial P_{i} / \partial \sigma_{j}\right)_{E_{i}}$ in the following form:

$$
d_{3 i}^{T}=\sum_{k=1}^{3} s_{i k}^{T E} e_{3 k}^{T}+s_{i 5}^{T E} e_{35}^{T}, \quad d_{35}^{T}=s_{15}^{T E} e_{31}^{T}+s_{25}^{T E} e_{32}^{T}+s_{35}^{T E} e_{33}^{T}+s_{55}^{T E} e_{35}^{T},
$$

where $s_{i k}^{E}=\left(\partial \varepsilon_{i} / \partial \sigma_{k}\right)_{E_{1}}, s_{j j}^{E}=\left(\partial \varepsilon_{j} / \partial \sigma_{j}\right)_{E_{i}}$ are the compliances at the constant field.

Using relations (3.3) one can obtain the expression for the static dielectric permittivity of a free RHS crystal

$$
\chi_{33}^{T \sigma}=\left(\frac{\partial P_{3}}{\partial E_{3}}\right)_{\sigma_{6}}=\chi_{23}^{T \varepsilon}+e_{31}^{T} d_{31}^{T}+e_{32}^{T} d_{32}^{T}+e_{33}^{T} d_{33}^{T}+e_{35}^{T} d_{35}^{T} .
$$

Molar entropy of RHS caused by its pseudospin subsystem is as follows:

$$
S=-R\left(\frac{\partial g}{\partial T}\right)=R\left[4 \ln 2+\sum_{f=1}^{4} \ln \cosh \frac{\beta}{2} \mathscr{H}_{f}-2 \gamma_{1} \xi_{1}-2 \gamma_{2} \xi_{2}-2 \gamma_{3} \xi_{3}-2 \delta \varsigma\right],
$$

where $R$ is the universal gas constant. Molar heat capacity at a constant pressure is calculated by differentiating the entropy 3.8

$$
\Delta C^{\sigma}=T\left(\frac{\partial S}{\partial T}\right)_{\sigma}
$$

\section{Relaxation dynamics of RHS crystal}

This section describes the dynamic phenomena in RHS at the application of electrical field $E_{1}^{*}$ to a crystal. While calculating the dynamic characteristics, we use the kinetic equation [25, 26] based on the Zubarev nonequilibrium statistical operator method [27].

The kinetic equation for the mean values of pseudospin operator is as follows:

$$
\frac{\mathrm{d}}{\mathrm{d} t}\left\langle\hat{p}_{m}\right\rangle=-\sum_{q f} \sum_{\mu \alpha}\left[Q_{q f \mu \alpha}^{-}\left(\hat{p}_{m}\right)+\tanh \frac{\beta \Omega_{\mu}^{\alpha}}{2} Q_{q f \mu \alpha}^{+}\left(\hat{p}_{m}\right)\right] K_{\mu}^{\alpha},
$$


where

$$
\begin{aligned}
& Q_{q f \mu \alpha}^{\mp}\left(\hat{p}_{m}\right)=\left\langle\left[\left[\hat{p}_{m}, \sigma_{q f}^{-\alpha}\left(\Omega_{\mu}^{\alpha^{\prime}}\right)\right], \sigma_{q f}^{\alpha}\left(\Omega_{\mu}^{\alpha}\right)\right]^{\mp}\right\rangle_{q}, \\
& K_{\mu}^{\alpha}=\int_{0}^{\infty} \mathrm{d} t \mathrm{e}^{-\varepsilon t} \cos \Omega_{\mu}^{\alpha} t \operatorname{Re}\left\langle\bar{u}(t) \bar{u}^{+}\right\rangle_{q}, \quad \alpha=0, \pm 1,
\end{aligned}
$$

while $\left\langle\bar{u}^{\alpha}(t) \bar{u}^{\alpha^{\prime}}\right\rangle_{q}$ is correlation function of the thermostat; $\sigma_{q f}^{\alpha}\left(\Omega_{\mu}^{\alpha}\right)$ is a Fourier component of the operator $\sigma_{q f}^{\alpha}(t) ; \Omega_{\mu}^{\alpha}$ are the eigenfrequencies of the Hamiltonian of the quasispin model $2.6 ; \sigma_{q f}^{0}=\sigma_{q f}$, $\sigma_{q f}^{ \pm}=\sigma_{q f}^{x} \pm i \sigma_{q f}^{y}$.

Taking into account (2.7), operators $\hat{p}_{m}$ have such a form:

$$
\hat{P}_{m}=\frac{\sigma_{q f}}{2} .
$$

Using the evolution law of the quasispin operators $S_{q f}^{\alpha}(\alpha=0 \pm)$ and their permutation relations, we calculate the commutators occurring in 4.2 as well as the expression for $Q_{q f \mu \alpha}^{\mp}\left(\hat{p}_{m}\right)$. The kinetic equation 4.1 can be rewritten as follows:

$$
-\frac{\mathrm{d}}{\mathrm{d} t} \eta_{f}=2 K_{f} \eta_{f}-2 K_{f} \tanh \frac{\beta H_{f}}{2},
$$

where

$$
K_{f}=\int_{0}^{\infty} \mathrm{d} t \mathrm{e}^{-\varepsilon t} \cos \left(H_{f} t\right) \operatorname{Re}\left[\left\langle\bar{u}^{-}(t) \bar{u}^{+}\right\rangle_{q}+\left\langle\bar{u}^{+}(t) \bar{u}^{-}\right\rangle_{q}\right] .
$$

Note that at $K_{f}=\frac{1}{2 \alpha}$, the obtained kinetic equation 4.5 agrees with the equation found within the stochastic Glauber model [28]. Using the variables $\xi_{1}, \xi_{2}, \xi_{3}, \zeta$ in equations [4.5], we obtain

$$
\begin{aligned}
& -\alpha \frac{\mathrm{d}}{\mathrm{d} t} \xi_{1}=\xi_{1}-\frac{1}{4}\left(-L_{1}-L_{2}+L_{3}+L_{4}\right), \\
& -\alpha \frac{\mathrm{d}}{\mathrm{d} t} \xi_{2}=\xi_{2}-\frac{1}{4}\left(-L_{1}-L_{2}-L_{3}-L_{4}\right), \\
& -\alpha \frac{\mathrm{d}}{\mathrm{d} t} \xi_{3}=\xi_{3}-\frac{1}{4}\left(L_{1}+L_{2}+L_{3}+L_{4}\right), \\
& -\alpha \frac{\mathrm{d}}{\mathrm{d} t} \zeta=\zeta-\frac{1}{4}\left(L_{1}-L_{2}+L_{3}-L_{4}\right),
\end{aligned}
$$

where the following notations are used:

$$
\begin{array}{rlrl}
L_{1} & =\tanh \frac{1}{2}\left(-\gamma_{1}-\gamma_{2}+\gamma_{3}+\delta\right), & L_{2} & =\tanh \frac{1}{2}\left(-\gamma_{1}+\gamma_{2}+\gamma_{3}-\delta\right), \\
L_{3} & =\tanh \frac{1}{2}\left(\gamma_{1}+\gamma_{2}+\gamma_{3}+\delta\right), & L_{4}=\tanh \frac{1}{2}\left(\gamma_{1}-\gamma_{2}+\gamma_{3}-\delta\right) .
\end{array}
$$

The dynamic properties RHS are explored using the system of equations (4.6) and at small deviations from the equilibrium. We separate these equations into the static and dynamic parts. The distribution functions are presented as sums of two components: the equilibrium functions and their deviations from the equilibrium values (fluctuations)

$$
\xi_{1}=\tilde{\xi}_{1}+\xi_{i t}, \quad(i=1,2,3), \quad \zeta=\tilde{\zeta}+\zeta_{t} .
$$

Also $E_{i t}=E_{i 0} \mathrm{e}^{\mathrm{i} \omega t}$.

As a result, we obtain the following system of equations for the fluctuation parts:

$$
-\frac{\mathrm{d}}{\mathrm{d} t}\left(\begin{array}{l}
\xi_{1 t s}(1) \\
\xi_{2 t s}(1)
\end{array}\right)=\left(\begin{array}{ll}
a_{11} & a_{12} \\
a_{21} & a_{22}
\end{array}\right)\left(\begin{array}{c}
\xi_{1 t s}(1) \\
\xi_{2 t s}(1)
\end{array}\right)-\frac{\beta \mu_{1} E_{1 t}}{2}\left(\begin{array}{c}
a_{1} \\
a_{2}
\end{array}\right)
$$




$$
\begin{aligned}
& -\frac{\mathrm{d}}{\mathrm{d} t}\left(\begin{array}{c}
\xi_{2 t s}(2) \\
\xi_{1 t s}(2)
\end{array}\right)=\left(\begin{array}{ll}
b_{11} & b_{12} \\
b_{21} & b_{22}
\end{array}\right)\left(\begin{array}{c}
\xi_{2 t s}(2) \\
\xi_{1 t s}(2)
\end{array}\right)-\frac{\beta \mu_{2} E_{2 t}}{2}\left(\begin{array}{l}
b_{1} \\
b_{2}
\end{array}\right), \\
& -\frac{\mathrm{d}}{\mathrm{d} t}\left(\begin{array}{c}
\xi_{3 t s}(3) \\
\zeta_{s t}(3)
\end{array}\right)=\left(\begin{array}{ll}
c_{11} & c_{12} \\
c_{21} & c_{22}
\end{array}\right)\left(\begin{array}{c}
\xi_{3 t s}(3) \\
\zeta_{s t}(3)
\end{array}\right)-\frac{\beta \mu_{3} E_{3 t}}{2}\left(\begin{array}{c}
c_{1} \\
c_{2}
\end{array}\right) .
\end{aligned}
$$

Solving the systems (4.9)-(4.11), we find the dynamic permittivities of the clamped RHS crystal

$$
\varepsilon_{i i s}^{\varepsilon}(\omega)=\varepsilon_{i i s}^{\varepsilon 0}+\sum_{j=1}^{2} \frac{4 \pi \chi_{j i s}}{1+\left(\omega \tau_{j i s}\right)^{2}}+i \sum_{j=1}^{2} \frac{4 \pi \omega \tau_{j i s} \chi_{j i s}}{1+\left(\omega \tau_{j i s}\right)^{2}} .
$$

In 4.12

$$
\begin{gathered}
\chi_{1 i s}=\frac{\mu_{i}^{2}}{v} \beta \frac{\tau_{1 i s} \tau_{2 i s}}{\tau_{1 i s}-\tau_{2 i s}}\left[-m^{(1)}(i)+\tau_{1 i s} m^{(0)}(i)\right], \quad \chi_{2 i s}=\frac{\mu_{i}^{2}}{v} \beta \frac{\tau_{1 i s} \tau_{2 i s}}{\tau_{1 i s}-\tau_{2 i s}}\left[m^{(1)}(i)-\tau_{2 i s} m^{(0)}(i)\right], \\
\tau_{\frac{1}{2} \text { is }}^{-1}=\frac{1}{2}\left[m_{1}(i) \mp \sqrt{m_{1}^{2}(i)-4 m_{0}(i)}\right] .
\end{gathered}
$$

In (4.13), we use the following notations:

$$
\begin{aligned}
& m_{1}(1)=m_{1}(2)=\frac{1}{\alpha}\left(1-\rho_{31} \frac{\beta J_{1}}{4}\right)+\frac{1}{\alpha}\left(1-\rho_{31} \frac{\beta J_{2}}{4}\right), \\
& m_{0}(1)=m_{0}(2)=\frac{1}{\alpha^{2}}\left[1-\rho_{31}\left(\frac{\beta J_{1}}{4}+\frac{\beta J_{2}}{4}\right)+\left(\rho_{31}^{2}-\rho_{32}^{2}\right) \frac{\beta J_{1}}{4} \frac{\beta J_{2}}{4}\right], \\
& m^{(0)}(1)=\frac{1}{\alpha^{2}}\left[\rho_{31}-\left(\rho_{31}^{2}-\rho_{32}^{2}\right) \frac{\beta J_{2}}{4}\right], \quad m^{(0)}(2)=\frac{1}{\alpha^{2}}\left[\rho_{31}-\left(\rho_{31}^{2}-\rho_{32}^{2}\right) \frac{\beta J_{1}}{4}\right] . \\
& m_{1}(3)=\frac{1}{\alpha}\left(1-\rho_{31} \frac{\beta J_{3}}{4}\right)+\frac{1}{\alpha}\left(1-\rho_{31} \frac{\beta J_{4}}{4}\right), \\
& m_{0}(3)=\frac{1}{\alpha^{2}}\left[1-\rho_{31}\left(\frac{\beta J_{3}}{4}+\frac{\beta J_{4}}{4}\right)+\left(\rho_{31}^{2}-\rho_{32}^{2}\right) \frac{\beta J_{3}}{4} \frac{\beta J_{4}}{4}\right], \\
& m^{(1)}(3)=\frac{1}{\alpha} \rho_{31}, \quad m^{(0)}(3)=\frac{1}{\alpha^{2}}\left[\rho_{31}-\left(\rho_{31}^{2}-\rho_{32}^{2}\right) \frac{\beta J_{4}}{4}\right] .
\end{aligned}
$$

\section{Comparison of numerical results with experimental data}

To compare the temperature and field dependences of the above derived dielectric, piezoelectric, elastic, and thermal characteristics of RHS, we need to set the values of the following parameters: the interaction potentials $J^{0}, K_{12}^{0}, K_{13}^{0}, K_{14}^{0}$ and, accordingly, $J_{1}, J_{2}, J_{3}, J_{4}$; the parameter $\Delta$, which characterizes an asymmetry of populations of the two equilibrium positions of a dipole; the deformation potentials $\psi_{i j}$; effective dipole moments $\mu_{i}$; seed dielectric permittivities $\chi_{i i}^{\varepsilon 0}$; piezoelectric coefficients $e_{i j}^{0}$; elastic constants $c_{i i^{\prime}}^{E 0}$ and $c_{j j}^{E 0}$, and the parameter $\alpha$ that defines the time scale of the relaxation processes.

To find the optimum values of the theory parameters it is necessary to use the dependence of temperature $T_{\mathrm{c}}$ on hydrostatic pressure. Unfortunately, different sources give different values for $T_{\mathrm{c}}(0)$, varying from $258 \mathrm{~K}$ to $265.1 \mathrm{~K}$. We shall use $T_{\mathrm{c}}(0)=265 \mathrm{~K}$ [14].

In the fitting procedure, we use the experimentally obtained values for the temperature dependences of the following physical characteristics of RHS: $P_{S}(T)$ [20], $\varepsilon_{11}^{\sigma}(0), \varepsilon_{22}^{\sigma}(0)$ [23], $\varepsilon_{33}(\omega)$ [14], as well as the dependence $T_{\mathrm{c}}(p)$ [29] of the transition temperature on hydrostatic pressure. In the case of deuterted RDS crystal, we exploit $P_{s}(T)$ [16], $\varepsilon_{33}^{\sigma}(0)$ [16], $T_{\mathrm{c}}(p)$ [29].

In order to find the values of the parameters $J^{0}+K_{13}^{0}, K_{12}^{0}+K_{14}^{0}, \Delta$, we found the point at the phase diagram $(a, b)$, where

$$
a=\frac{\left(J^{0}+K_{13}^{0}\right)-\left(K_{12}^{0}+K_{14}^{0}\right)}{\left(J^{0}+K_{13}^{0}\right)+\left(K_{12}^{0}+K_{14}^{0}\right)}, \quad b=\frac{\Delta}{\left(J^{0}+K_{13}^{0}\right)+\left(K_{12}^{0}+K_{14}^{0}\right)},
$$


at which the system undergoes a single second order phase transition at $T_{\mathrm{c}}(0)=265 \mathrm{~K}$. The values of the effective dipole moments $\mu_{3}$ were determined by fitting the calculated spontaneous polarization to experiment.

The values of $J^{0}, K_{12}^{0}, K_{13}^{0}, K_{14}^{0}, \mu_{1}$, and $\mu_{2}$ are determined by fitting the calculated $\varepsilon_{11}$ and $\varepsilon_{22}$ to the experimental data given in [23].

The fitting procedure for the models of this class is described in detail in [30], where the thermodynamic characteristics of Rochelle salt are explored.

The strains should be taken into account in order to calculate the dielectric permittivity of a mechanically free crystal, piezoelectric coefficients, and elastic constants. Therefore, to determine the values of the deformation potentials $\psi_{i j}$, we analyzed their effect on theoretical values of physical characteristics of the crystal. Thus, it has been obtained that an increase of the transition temperature with hydrostatic pressure [29] can be described using the values of $\psi_{i j}$ presented here. It should be stressed that when the piezoelectric coefficients are measured experimentally, the values of $\psi_{i j}$ can be determined with a greater accuracy.

The parameter $\alpha_{H}$ is determined from the condition that the theoretically calculated frequency dependences of $\varepsilon_{33}(\omega)$ agree with the experiment. We also assume that the parameter $\alpha_{H}$ is a weak function of temperature:

$$
\alpha_{H}=\left[P_{H}+R_{H}(\Delta T)\right] \times 10^{-14}, \quad \Delta T=T-T_{\mathrm{c}} .
$$

The unit cell volume of RHS is $v=0.842 \cdot 10^{-21} \mathrm{~cm}^{3}$.

The obtained sets of optimal parameters are given in table 1

Table 1. The optimal sets of the theory parameters for $\mathrm{Rb}\left(\mathrm{H}_{1-x} \mathrm{D}_{x}\right) \mathrm{SO}_{4}$.

\begin{tabular}{|c|c|c|c|c|c|c|c|c|c|c|c|}
\hline \hline$x$ & $\begin{array}{c}J^{0} / k_{\mathrm{B}} \\
\mathrm{K}\end{array}$ & $\begin{array}{c}K_{12}^{0} / k_{\mathrm{B}} \\
\mathrm{K}\end{array}$ & $\begin{array}{c}K_{13}^{0} / k_{\mathrm{B}} \\
\mathrm{K}\end{array}$ & $\begin{array}{c}K_{14}^{0} / k_{\mathrm{B}} \\
\mathrm{K}\end{array}$ & $\begin{array}{c}\Delta / k_{\mathrm{B}} \\
\mathrm{K}\end{array}$ & $\begin{array}{c}\mu_{1}, 10^{-18} \\
\mathrm{esu} \cdot \mathrm{cm}\end{array}$ & $\begin{array}{c}\mu_{2}, 10^{-18} \\
\mathrm{esu} \cdot \mathrm{cm}\end{array}$ & $\begin{array}{c}\mu_{3}, 10^{-18} \\
\text { esu·cm }\end{array}$ & $\chi_{11}^{\varepsilon 0}$ & $\chi_{22}^{\varepsilon 0}$ & $\chi_{33}^{\varepsilon 0}$ \\
\hline 0.0 & 394 & 190 & 372 & 433.7 & 244 & 3.18 & 3.65 & 0.81 & 0.02 & 0.02 & 0.159 \\
\hline 0.7 & 380 & 189 & 345 & 430.8 & 245 & & & 0.90 & 0.02 & 0.02 & 0.159 \\
\hline 1.0 & 378 & 198.8 & 338.4 & 429 & 245.4 & & & 1.00 & 0.02 & 0.02 & 0.159 \\
\hline \hline
\end{tabular}

\begin{tabular}{|c|c|c|c|c|c|c|}
\hline \hline$x$ & $\begin{array}{c}P_{3 s} \\
(\mathrm{~s})\end{array}$ & $\begin{array}{c}R_{3 s} \\
(\mathrm{~s} / \mathrm{K})\end{array}$ & $\begin{array}{c}P_{3 p} \\
(\mathrm{~s})\end{array}$ & $\begin{array}{c}P_{3 p} \\
(\mathrm{~s} / \mathrm{K})\end{array}$ & $\begin{array}{c}P_{1,2} \\
(\mathrm{~s})\end{array}$ & $\begin{array}{c}R_{1,2} \\
(\mathrm{~s} / \mathrm{K})\end{array}$ \\
\hline 0.0 & 12.5 & -0.0521 & 12.5 & -0.091 & 10.5 & -0.001 \\
\hline 0.7 & 10.7 & -0.0510 & 10.7 & -0.051 & & \\
\hline 1.0 & 9.8 & -0.0510 & 9.8 & -0.0501 & & \\
\hline \hline
\end{tabular}

The deformation potentials are taken to be $\bar{\psi}_{1 i} / k_{\mathrm{B}}=\bar{\psi}_{1 j} / k_{\mathrm{B}}=\bar{\psi}_{2 i} / k_{\mathrm{B}}=\bar{\psi}_{2 j} / k_{\mathrm{B}}=900 \mathrm{~K}, \bar{\psi}_{31} / k_{\mathrm{B}}=$ $-4950 \mathrm{~K}, \bar{\psi}_{32} / k_{\mathrm{B}}=\bar{\psi}_{33} / k_{\mathrm{B}}=\bar{\psi}_{35} / k_{\mathrm{B}}=-4500 \mathrm{~K}, \bar{\psi}_{41} / k_{\mathrm{B}}=1080 \mathrm{~K}, \bar{\psi}_{42} / k_{\mathrm{B}}=\bar{\psi}_{43} / k_{\mathrm{B}}=\bar{\psi}_{45} / k_{\mathrm{B}}=900 \mathrm{~K}$, $\bar{\psi}_{51} / k_{\mathrm{B}}=\bar{\psi}_{52} / k_{\mathrm{B}}=\bar{\psi}_{53} / k_{\mathrm{B}}=\bar{\psi}_{55} / k_{\mathrm{B}}=200 \mathrm{~K}$.

The "seed" constants for RHS are $e_{31}^{0}=e_{32}^{0}=e_{33}^{0}=e_{35}^{0}=-1 \times 10^{4} \mathrm{esu} / \mathrm{cm}^{2}, c_{11}^{0 E}=32.0 \times 10^{10} \mathrm{dyn} / \mathrm{cm}^{2}$, $c_{12}^{E 0}=17.0 \times 10^{10} \mathrm{dyn} / \mathrm{cm}^{2}, c_{13}^{E 0}=8.7 \times 10^{10} \mathrm{dyn} / \mathrm{cm}^{2}, c_{22}^{E 0}=38 \times 10^{10} \mathrm{dyn} / \mathrm{cm}^{2}, c_{23}^{E 0}=6.5 \times 10^{10} \mathrm{dyn} / \mathrm{cm}^{2}$, $c_{33}^{E 0}=37.4 \times 10^{10} \mathrm{dyn} / \mathrm{cm}^{2}, c_{44}^{E 0}=4.9 \times 10^{10} \mathrm{dyn} / \mathrm{cm}^{2}, c_{55}^{E 0}=5.3 \times 10^{10} \mathrm{dyn} / \mathrm{cm}^{2}, c_{66}^{E 0}=12.8 \times 10^{10} \mathrm{dyn} / \mathrm{cm}^{2}$; $k_{11}=-0.032 \times 10^{10} \mathrm{dyn} /\left(\mathrm{cm}^{2} \mathrm{~K}\right), k_{12}=-0.040 \times 10^{10} \mathrm{dyn} /\left(\mathrm{cm}^{2} \mathrm{~K}\right), k_{13}=-0.015 \times 10^{10} \mathrm{dyn} /\left(\mathrm{cm}^{2} \mathrm{~K}\right), k_{23}=$ $-0.010 \times 10^{10} \mathrm{dyn} /\left(\mathrm{cm}^{2} \mathrm{~K}\right) k_{33}=-0.032 \times 10^{10} \mathrm{dyn} /\left(\mathrm{cm}^{2} \mathrm{~K}\right), k_{22}=k_{44}=k_{55}=0.0$. We use the same values for RDS as well.

Now we discuss the obtained results. In figure 2 the temperature dependences of the strains $\varepsilon_{i}$ and $\varepsilon_{j}$ are presented. In the ferroelectric phase, $\varepsilon_{i}$ slightly increases with temperature, while the temperature variation of $\varepsilon_{4}, \varepsilon_{6}$, and especially $\varepsilon_{5}$ is much stronger. In the paraelectric phase, all these strains weakly increase with temperature. The temperature dependences of spontaneous polarization $P_{s}$ of RHS and RDS along with the experimentally obtained values [16, 17, 20] are shown in figure 3, A good description of experimental data of [20] and [16] is reached. When the deuteration level $x$ increases, the polarization decreases. 


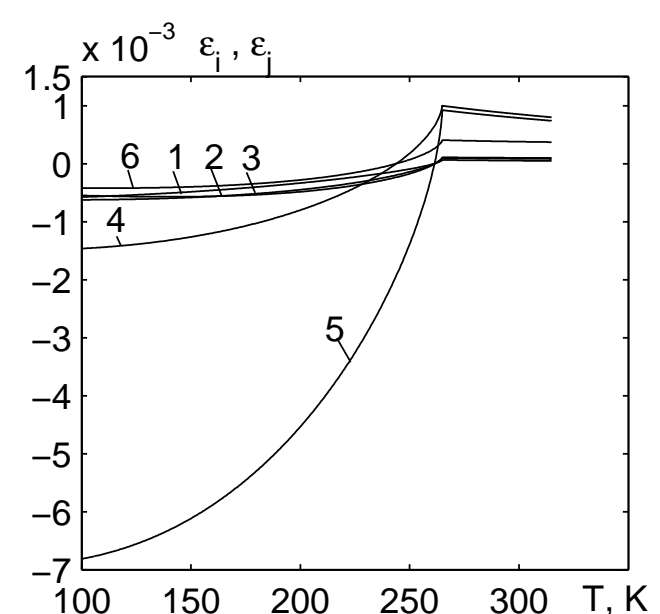

Figure 2. The temperature dependences of the strains $\varepsilon_{i}$ and $\varepsilon_{j}$ of RHS: $\varepsilon_{1}-1, \varepsilon_{2}-2, \varepsilon_{3}-3, \varepsilon_{4}-$ $4, \varepsilon_{5}-5$ i $\varepsilon_{6}-6$.

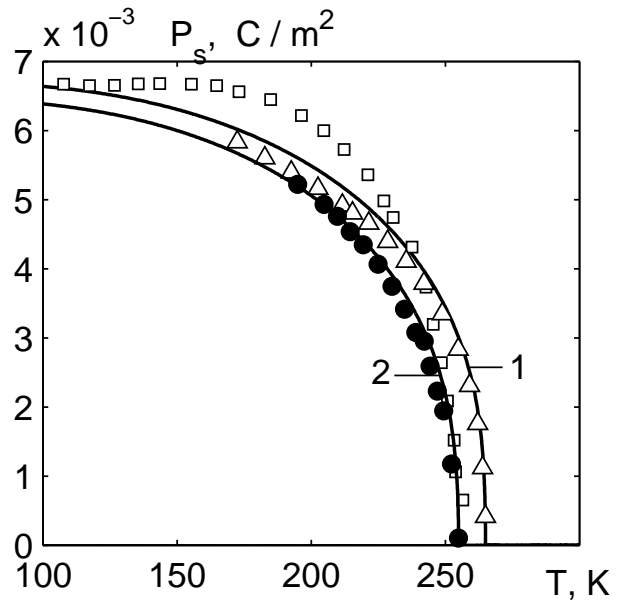

Figure 3. The temperature dependences of spontaneous polarization of RHS $-1, \Delta[20], \square[17]$ and $\mathrm{RDS}-2, \bullet[16$.

Figure 4 shows the calculated temperature dependences of static dielectric permittivities of the mechanically clamped $\varepsilon_{33}^{\varepsilon}(0, T)$ and free $\varepsilon_{33}^{\sigma}(0, T)$ RHS crystal along with the experimental data [17, 19 [20. 23.

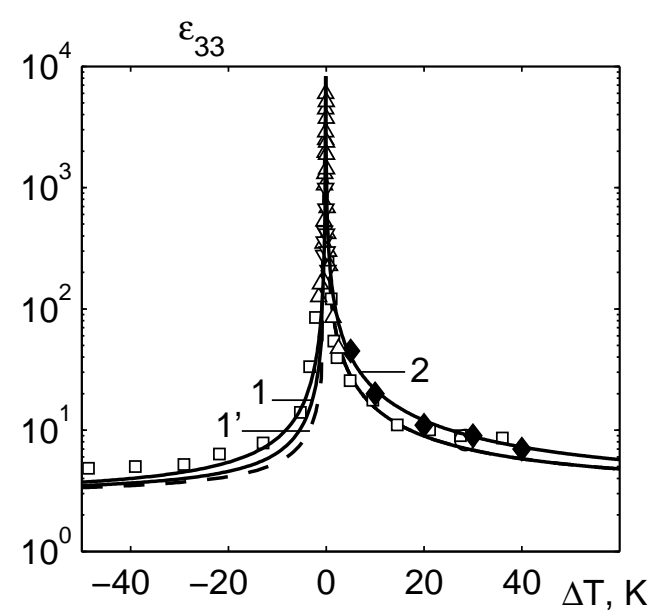

Figure 4. The temperature dependences of static dielectric permittivities of RHS, $1-\varepsilon_{33}^{\sigma}, 1^{\prime}-\varepsilon_{33}^{\varepsilon}$, $\nabla$ [19], $\Delta$ [20], $\square$ [17], $\circ$ [23] and RDS $-2, \diamond[16]$.

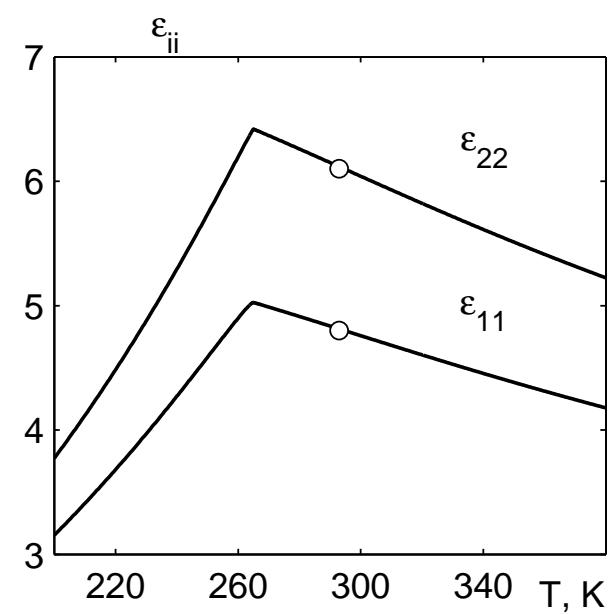

Figure 5. The temperature dependences of the transverse permittivities $\varepsilon_{11}$ and $\varepsilon_{22}$ of RHS. $\circ$ [23].

The permittivity $\varepsilon_{33}^{\sigma}(0, T)$ is larger than $\varepsilon_{33}^{\varepsilon}(0, T)$. An increase of deuteron concentration increases the permittivity $\varepsilon_{33}^{\varepsilon}(0, T)$ at all temperatures. As shown in figure 4 the theoretical results $\varepsilon_{33}^{\sigma}(0, T)$ are in a good quantitative agreement with experimental data of [17, 19, 20, 23]. At temperature $T=T_{\mathrm{c}}$, the value of the permittivity $\varepsilon_{33}^{\sigma}(0, T)$ is very large, which is typical of the second order phase transitions.

Figure 5 illustrates the temperature dependences of the transverse permittivities $\varepsilon_{11}$ and $\varepsilon_{22}$ of a RHS crystal. They are significantly smaller than the longitudinal permittivity.

The temperature dependences of piezoelectric coefficients $e_{3 i}$ and $e_{55}$ and constants $h_{3 i}$ and $h_{35}$ are given in figure 6 In the paraelectric phase, these coefficients are equal to zero, whereas in the ferroelectric phase, $e_{3 i}$ and $e_{55}$ values have a deep minimum at approaching $T_{\mathrm{c}}$, and $h_{31}, h_{35}$ constants change insignificantly. 

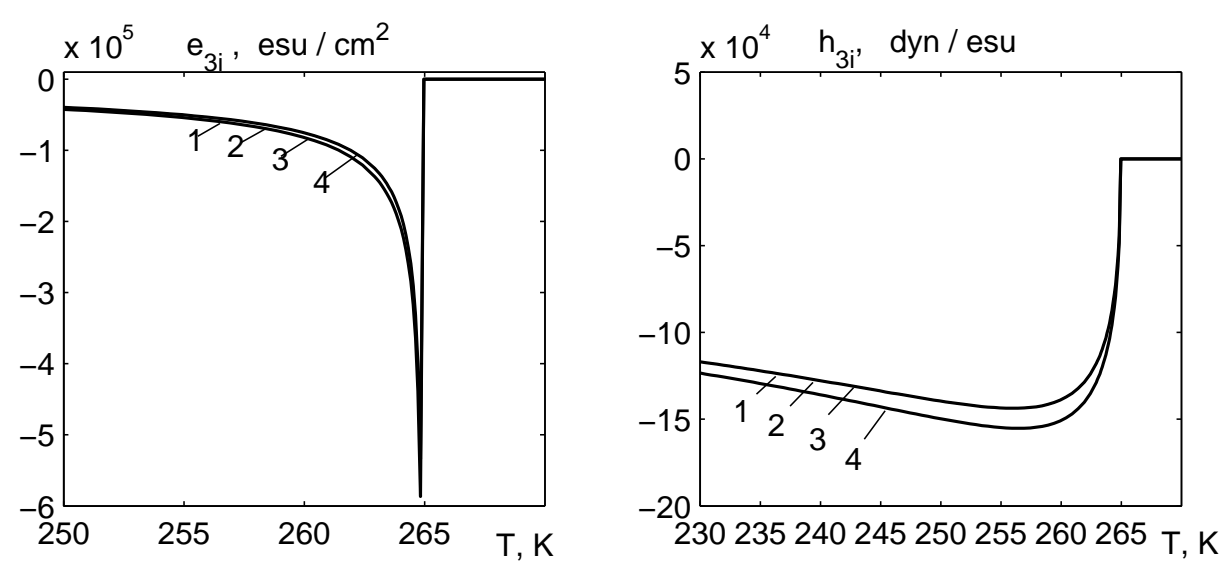

Figure 6. The temperature dependences of piezoelectric coefficients $e_{31}-1, e_{32}-2, e_{33}-3, e_{35}-4$ and constants $h_{31}-1, h_{32}-2, h_{33}-3, h_{35}-4$ of RHS.

In figure 7 we show the temperature dependences of the elastic constants $c_{i j}^{E}$. A good description of experimental data [23] is obtained, except for the temperature dependence of elastic constants $c_{12}^{E}$, $c_{22}^{E}$, and $c_{23}^{E}$ in the ferroelectric phase, for which the experimental measurements predict deep minima near $T_{\mathrm{c}}$.

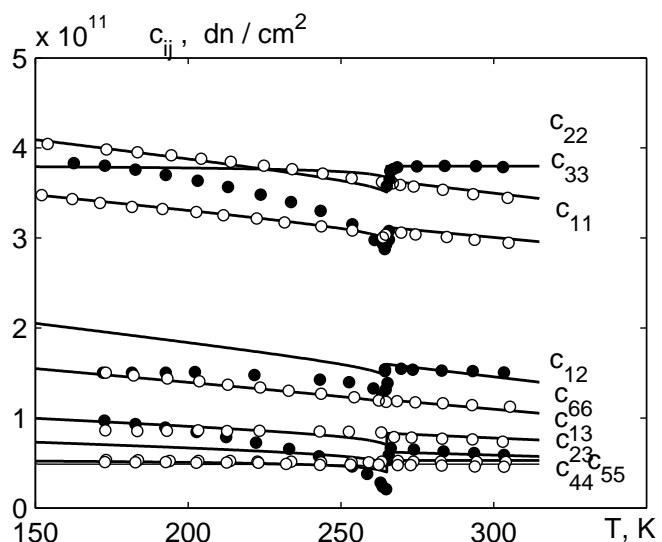

Figure 7. The temperature dependences of the elastic constants $c_{i j}^{E}$ of RHS, o- [23], lines: the theory.

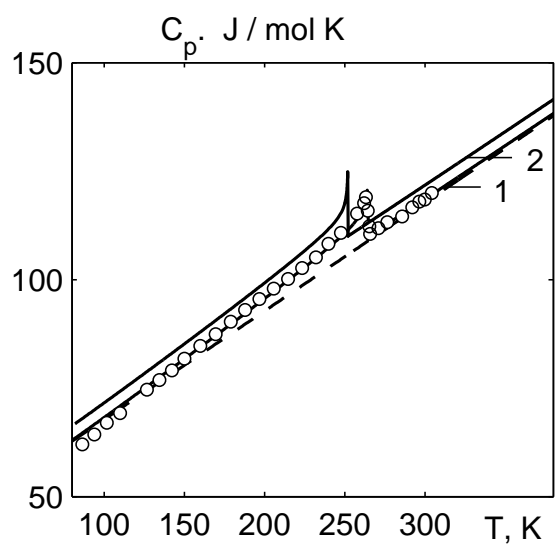

Figure 8. The temperature dependences of heat capacity of RHS $-1, \bullet[31$ and RDS -2 .

The temperature dependences of heat capacity of RHS and RDS crystals along with experimental data [31] are depicted in figure 8

By a dashed line we show the effective lattice heat capacity contribution $C_{0}$, which we estimate as an average difference $C_{\exp }(T)-\Delta C(T)$. A quantitatively good description of experiment [31] is obtained. The calculated value of the heat capacity jump is also in a good agreement with experiment. Deuteration increases the heat capacity in the entire temperature range.

Figures 911 show the temperature dependences of the real $\varepsilon_{33}^{\varepsilon^{\prime}}(v, T)$ and imaginary $\varepsilon_{33}^{\varepsilon^{\prime \prime}}(v, T)$ parts of the dynamic dielectric permittivity at different frequencies and compositions of partially deuterated $\mathrm{Rb}\left(\mathrm{H}_{1-x} \mathrm{D}_{x}\right) \mathrm{SO}_{4}$ crystals (at $x=0.0,0.70,1.00$ ) along with the experimental data [14 32, 35].

As seen in the figures, the proposed model yields a good description of experimental data for the $\mathrm{Rb}\left(\mathrm{H}_{1-x} \mathrm{D}_{x}\right) \mathrm{SO}_{4}$ crystals over a wide temperature range at different frequencies. For all frequencies, at $\Delta T=0 \mathrm{~K}$, the dynamic permittivity $\varepsilon_{33}^{\prime}(v, T)$ has a sharp minimum, where the permittivity values drop to $\varepsilon_{33}^{0 \varepsilon}$; the minimum width increases with an increasing frequency. The maximum in the temperature curve of $\varepsilon_{33}^{\prime}(v, T)$ above $T_{\mathrm{c}}$ lowers down, smears out, and shifts to higher temperatures at an increasing 

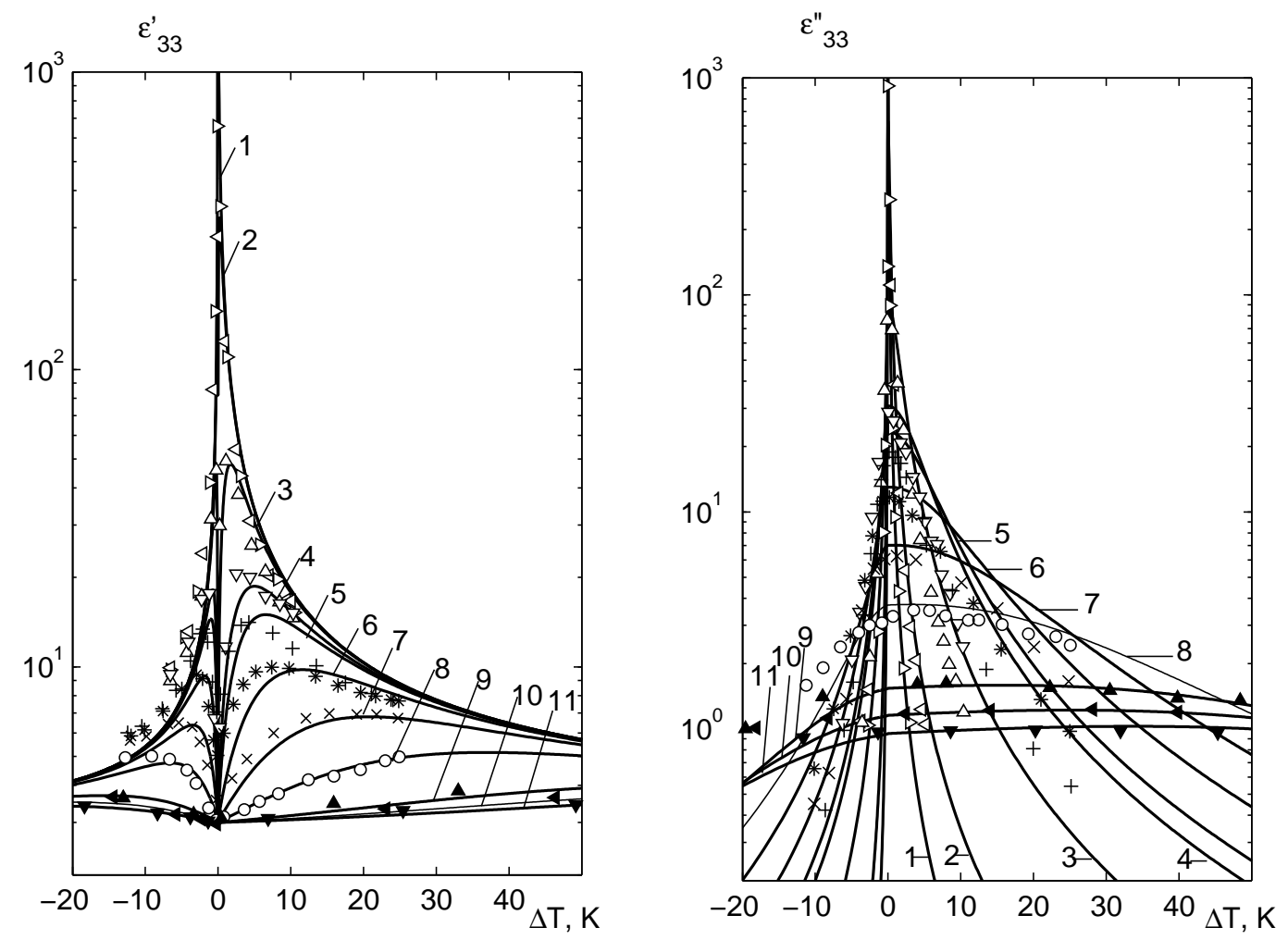

Figure 9. The temperature dependences of the real $\varepsilon_{33}^{\varepsilon^{\prime}}(v, T)$ and imaginary $\varepsilon_{33}^{\varepsilon^{\prime \prime}}(v, T)$ parts of the dynamic dielectric permittivity of RHS at different frequencies $v$ (GHz): $0.150-1, \triangleright$ [32]; $0.455-2, \triangleleft[32$ ]; $3.27-$ 3, $\Delta$ [32]; $9.50-4, \nabla$ [32], 8.72-5, + [14]; $12.5-6, *$ [14]; $22.5-7, \times$ [14]; $41.7-8, \circ$ [14]; $190-9, \Delta$ [35]; $253-10,4$ 35]; $307-11, \nabla$ [35.
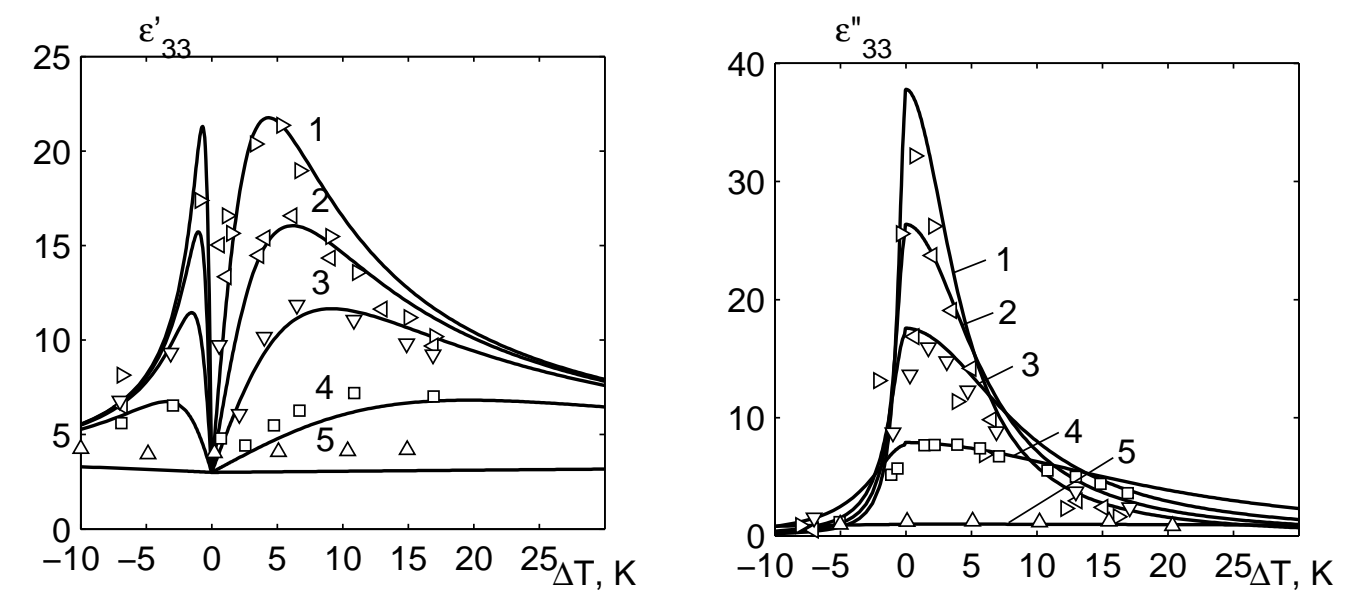

Figure 10. The temperature dependences of the real $\varepsilon_{33}^{\prime}$ and imaginary $\varepsilon_{33}^{\prime \prime}$ parts of the dynamic dielectric permittivity of $\mathrm{Rb}\left(\mathrm{H}_{0.30} \mathrm{D}_{0.70}\right) \mathrm{SO}_{4}$ at different frequencies $v(\mathrm{GHz}): 8.72-1$, $\triangleright$ [14]; $12.5-2, \triangleleft$ [14]; $18.72-3, \nabla$ [14]; $41.70-4, \square[14] ; 330-5, \Delta[14]$.

frequency. The dispersion width of the real part of the permittivity in the paraelectric phase is wider than in the ferroelectric phase.

In figures 12 and 13 we plot the temperature dependences of the real and imaginary parts of the transverse dielectric permittivities $\varepsilon_{11}^{\prime}, \varepsilon_{11}^{\prime \prime}$ and $\varepsilon_{22}^{\prime}, \varepsilon_{22}^{\prime \prime}$ of RHS at different frequencies. 

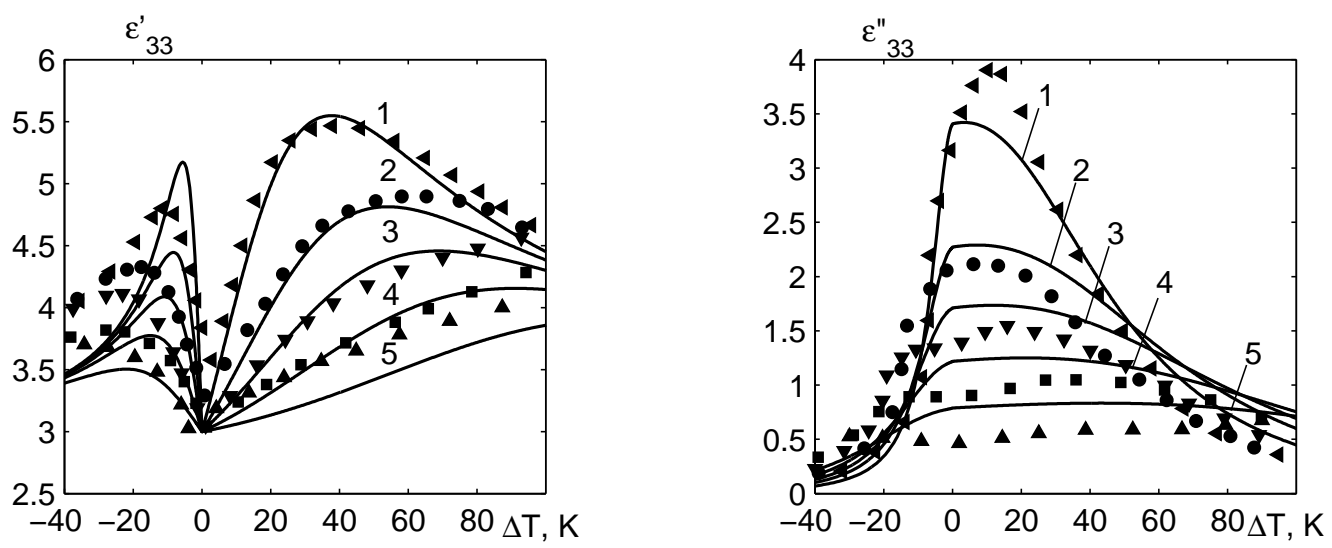

Figure 11. The temperature dependences of the real $\varepsilon_{33}^{\prime}$ and imaginary $\varepsilon_{33}^{\prime \prime}$ parts of the dynamic dielectric permittivity of RDS at different frequencies $v(\mathrm{GHz}): 118-1, \triangleleft[35] ; 177-2, \bullet[35] ; 235-3, \mathbf{\nabla}$ [35]; $330-$ 4, [35]; $510-5, \mathbf{\Delta}$ [35.
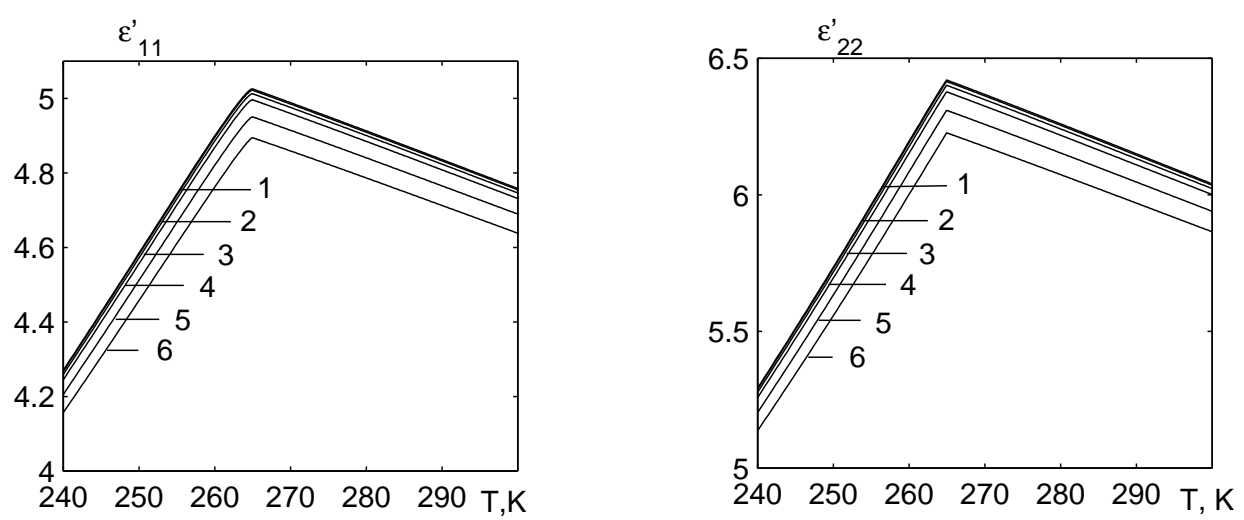

Figure 12. The temperature dependences of the real $\varepsilon_{11}^{\prime}$ and $\varepsilon_{22}^{\prime}$ parts of the dynamic dielectric permittivity of RDS at different frequencies $v(\mathrm{GHz}): 0-1 ; 41.7-2 ; 78.5-3 ; 118-4 ; 190-5 ; 253-6$.
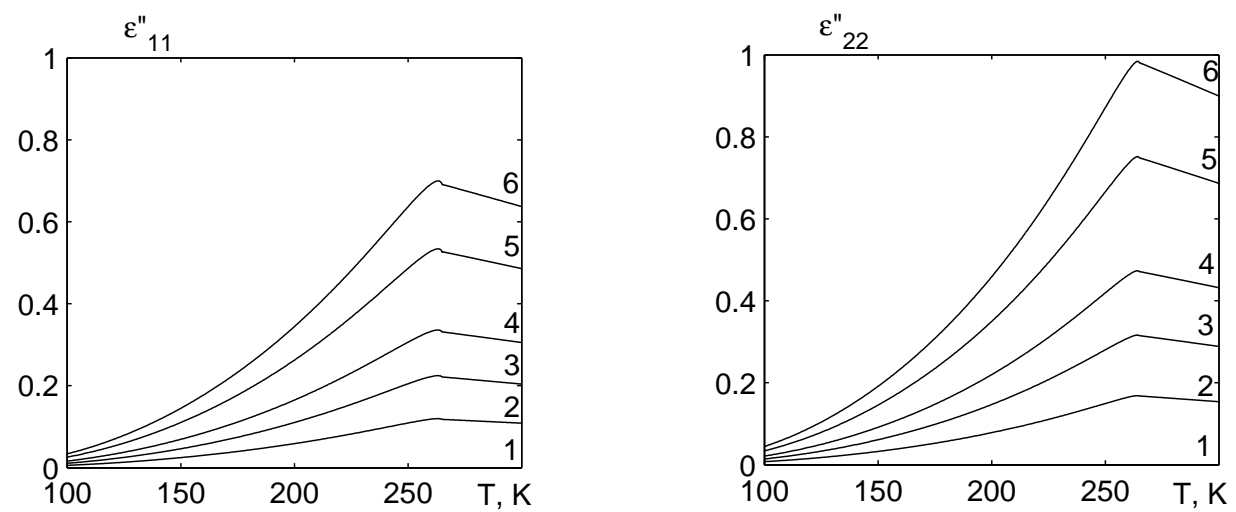

Figure 13. The temperature dependences of the imaginary $\varepsilon_{11}^{\prime \prime}$ and $\varepsilon_{22}^{\prime \prime}$ parts of the dynamic dielectric permittivity of RDS at different frequencies $v(\mathrm{GHz}): 0-1 ; 41.7-2 ; 78.5-3 ; 118-4 ; 190-5 ; 253-6$.

In figure 14 we plot the calculated frequency dependences of $\varepsilon_{33}^{\prime}(v)$ and $\varepsilon_{33}^{\prime \prime}(v)$ for RHS at different temperatures $\Delta T=2,5,10,20 \mathrm{~K}$ and in figure 15 for $\mathrm{Rb}\left(\mathrm{H}_{1-x} \mathrm{D}_{x}\right)_{2} \mathrm{SO}_{4}$ with $x=0.70$ along with the experimental points. 

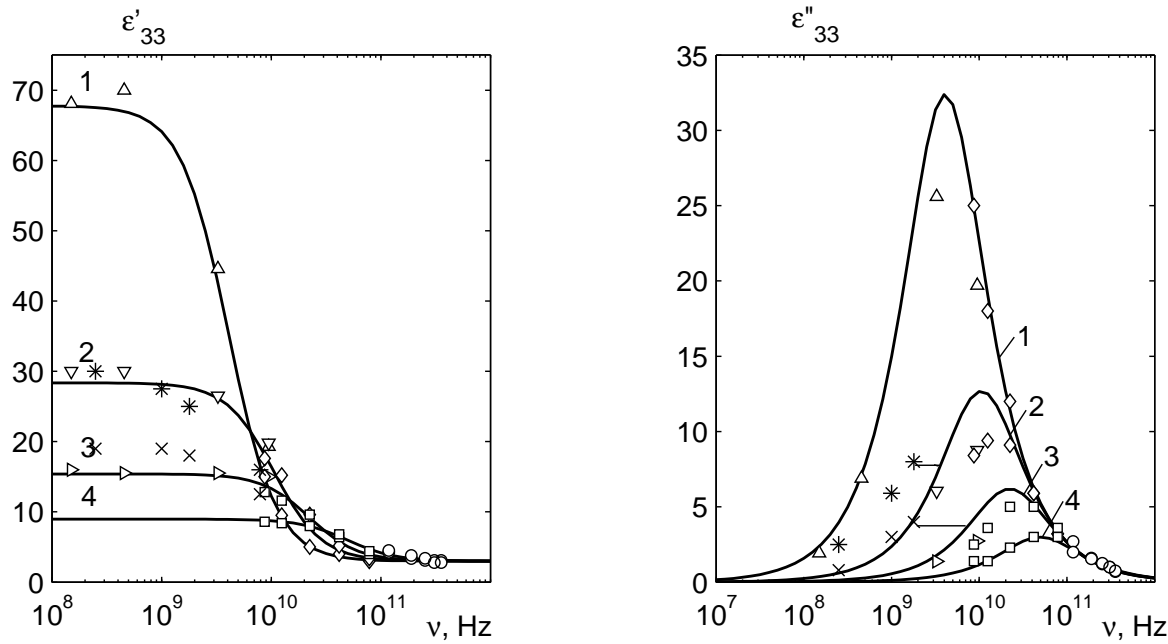

Figure 14. The frequency dependences of $\varepsilon_{33}^{\prime}(v)$ and $\varepsilon_{33}^{\prime \prime}(v)$ for RHS at $\Delta T: 2-1 ; 5-2 ; 10-3 ; 20-4$. $\Delta$ [32]; • [33]; ○ [14]; $\square[35], \times, *$ [33].
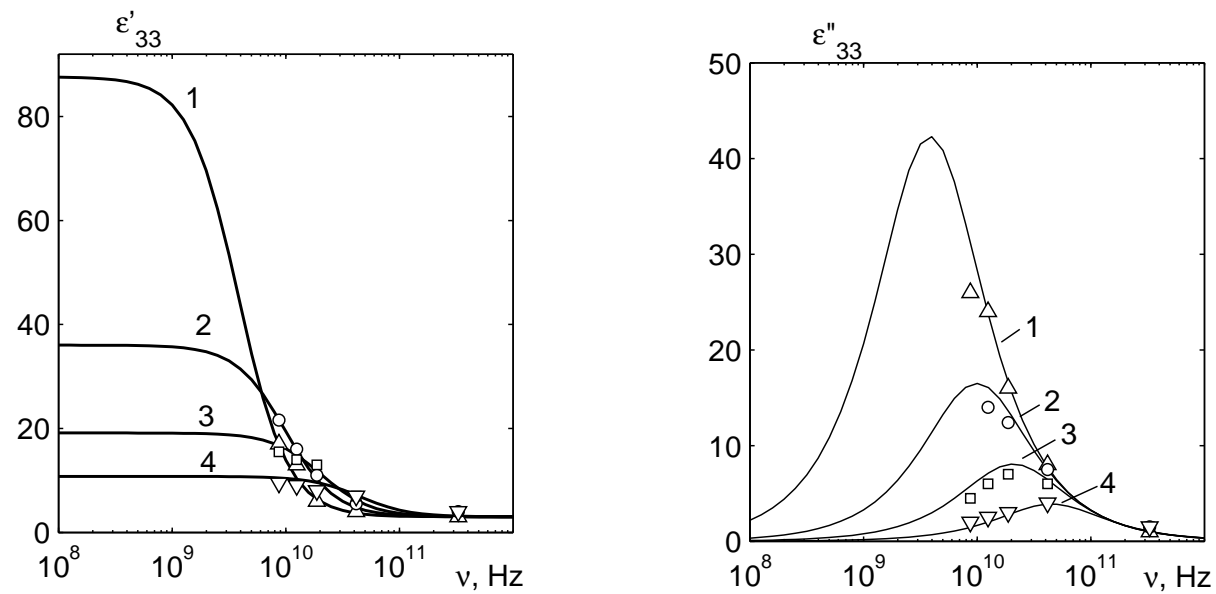

Figure 15. The frequency dependences of $\varepsilon_{33}^{\prime}(v)$ and $\varepsilon_{33}^{\prime \prime}(v)$ for $\mathrm{Rb}\left(\mathrm{H}_{1-x} \mathrm{D}_{x}\right)_{2} \mathrm{SO}_{4}$ at $x=0.70$ at $\Delta T: 2-1$, $\Delta$ [14]; $5-2$, ० [14]; $10-3, \square[14] ; 20-4, \nabla$ [14].

As one can see, the theory is in a good agreement with experiment, except for the data of [33] where the permittivity dispersion was observed at frequencies lower than in [14 32, 35]. When $\Delta T$ increases, the dispersion of $\varepsilon_{33}(v, T)$ shifts to higher frequencies.

\section{Conclusions}

In this paper, using the modified four-sublattice model of a $\mathrm{RbHSO}_{4}$ crystal, with taking into account the piezoelectric coupling to the $\varepsilon_{i}, \varepsilon_{j}$ strains, within the framework of the mean field approach, the theory of the thermodynamic, dielectric, piezoelectric, elastic, and dynamic properties of RHS crystals has been developed. A thorough numerical analysis of the dependences of the calculated characteristics on the model parameters has been performed. Optimal sets of these parameters and "seed" characteristics for RHS crystals have been found which enabled us to describe the available experimental data. 


\section{References}

1. Nelmes R.J., Ferroelectrics, 1972, 4, 133; doi 10.1080/00150197208235754

2. Ashmore J.P., Petch H.E., Can. J. Phys., 1975, 53, 2694; doi 10.1139/p75-328.

3. Iton K., Ohno H., Kuragaki S., J. Phys. Soc. Jpn., 1995, 64, 479; doi 10.1143/JPSJ.64.479

4. Iton K., Moriyoshi C., Ferroelectrics, 2003, 285, 91; doi 10.1080/00150190390205933

5. Kasahara M., Tatsuzaki I., J. Phys. Soc. Jpn., 1974, 36, 786; doi 10.1143/JPSJ.36.786

6. Kasahara M., Tatsuzaki I., J. Phys. Soc. Jpn., 1974, 37, 167; doi 10.1143/JPSJ.37.167

7. Kasahara M., Tatsuzaki I., J. Phys. Soc. Jpn., 1975, 38, 1389; doi 10.1143/JPSJ.38.1389

8. Mjasnikova E.P., Yacenko A.F., Fiz. Tverd. Tela, 1962, 4, 653 (in Russian).

9. Bazhulyn P.A., Mjasnikova E.P., Rarov A.V., Fiz. Tverd. Tela, 1963, 5, 1784 (in Russian).

10. Nakamura E., Kajikawa H., J. Phys. Soc. Jpn., 1978, 44, 519; doi 10.1143/JPSJ.44.519

11. Blat D.H., Zinenko V.I., Fiz. Tverd. Tela, 1976, 18, 3588 (in Russian).

12. Levitsky R.R., Zachek I.R., Varanisky V.I., Fiz. Tverd. Tela, 1980, 22, 2750 (in Russian).

13. Paprotny W., Grigas J., Levitsky R.R., Kutny I.V., Krasikov V.S., Ferroelectrics, 1984, 61, 19; doi $10.1080 / 00150198408018932$.

14. Grigas J., Zacekas I., Krasikovas V., Kutnas I., Levickis R., Paprotnas V., Lietuvos Fizikos Rinkinys, 1984, 24, No. 6, 33.

15. Levitsky R.R., Andrusyk A.Ya., Preprint of the Institute for Condensed Matter Physics, ICMP-11-03U, Lviv, 2011 (in Ukrainian).

16. Ichikawa M., J. Phys. Soc. Jpn., 1979, 47, 1562; doi 10.1143/JPSJ.47.1562

17. Pepinsky R., Vedam K., Phys. Rev., 1960, 117, No. 3, 1502; doi 10.1103/PhysRev.117.1502

18. Nakamura E., Kajikawa H., Ozaki T., J. Phys. Soc. Jpn., 1977, 42, 1427; doi 10.1143/JPSJ.42.1427

19. Flerov I.N., Iskornev I.M., Fiz. Tverd. Tela, 1976, 18, 3666 (in Russian).

20. Kajikawa H., Ozaki T., Nakamura E., J. Phys. Soc. Jpn., 1977, 43, 937; doi 10.1143/JPSJ.43.937

21. Stasyuk I.V., Levitsky R.R., Moina A.P., Slivka O.G., Velychko O.V., Field and Deformational Effects in Complex Ferroelectric Compounds, Grazhda, Uzhgorod, 2009 (in Ukrainian).

22. Stasyuk I., Velychko O., Ferroelectrics, 2005, 316, 51; doi 10.1080/00150190590963138

23. Zajceva M.P., Shabanova L.A., Zherebcova L.I., Fiz. Tverd. Tela, 1979, 21, 2308 (in Russian).

24. Levitsky R.R., Lisnii B.M., Phys. Status Solidi B, 2004, 241, 1350; doi 10.1002/pssb.200301995

25. Berim G.O., Kessel A.R., Physica A, 1980, 101, No. 1, 112; doi 10.1016/0378-4371(80)90103-X

26. Berim G.O., Kessel A.R., Physica A, 1980, 101, No. 1, 127; doi 10.1016/0378-4371(80)90104-1

27. Zubarev D.N., Noneguilibrium Statistical Thermodynamics, Consultants Bureau, New York, 1974.

28. Glauber J., J. Math. Phys., 1963, 4, No. 2, 294; doi 10.1063/1.1703954

29. Gesi K., Ozawa K., J. Phys. Soc. Jpn., 1975, 38, 459; doi 10.1143/JPSJ.38.459

30. Levitsky R.R., Zachek I.R., Vdovych A.S., Stasyuk I.V., Condens. Matter Phys., 2009, 12, No. 2, 295; doi $10.5488 /$ CMP.12.2.295

31. Alexanrov K.S., Anistratov A.T., Blat D.X., Zherebtsova L.I., Zinenko V.I., Iskornev I.M., Melnikova S.V., Flerov I.N., Kirenski L.V., Ferroelectrics, 1976, 12, 191; doi $10.1080 / 00150197608241423$

32. Ozaki T., J. Phys. Soc. Jpn., 1980, 49, 234; doi 10.1143/JPSJ.49.234.

33. Krasikov V.S., Ushatkin K.F., Ogurcov C.V., Fiz. Tverd. Tela, 1979, 21, 617 (in Russian).

34. Krasikov V.S., Ogurcov C.V., Ushatkin K.F., Fiz. Tverd. Tela, 1981, 23, 3425 (in Russian).

35. Ambrazjavichene V.S., Volkov A.A., Kozlov G.V., Krasikov V.S., Krjukova E.B., Fiz. Tverd. Tela, 1983, 25, 1605 (in Russian). 


\title{
Статистична теорія термодинамічних та динамічних властивостей сегнетоелектрика $\mathrm{RbHSO}_{4}$
}

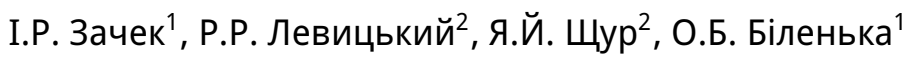 \\ 1 Національний університет “Львівська політехніка", вул. С. Бандери, 12, 79013 Львів, Україна \\ 2 Інститут фізики конденсованих систем НАН України, вул. І. Свєнціцького, 1, 79011 Львів, Україна
}

\begin{abstract}
Використовуючи модифіковану чотирипідграткову модель $\mathrm{RbHSO}_{4}$ шляхом врахування п'єзоелектричного зв'язку з деформаціями $\varepsilon_{i}, \varepsilon_{4}, \varepsilon_{5}$ і $\varepsilon_{6}$, в наближенні молекулярного поля розраховано компоненти вектора поляризації та тензора статичної діелектричної проникності механічно затиснутого і вільного кристалів, їх п'єзоелектричні характеристики і пружні сталі. Розраховано також динамічні проникності механічно затиснутого кристалу $\mathrm{RbHSO}_{4}$. При знайденому наборі параметрів теорії отримано для цих характеристик задовільний кількісний опис наявних експериментальних даних.
\end{abstract}

Ключові слова: сегнетоелектрики, діелектрична проникність, п'єзомодулі

PACS: 77.22.Ch, 77.22.Gm, 77.65.-j, 77.80.Bh, 77.84.-s 